\title{
CLOUD COMPUTING: A CROSS-CULTURAL COMPARATIVE STUDY BETWEEN COMPUTER AND INFORMATION SYSTEMS FACULTY AT A UNIVERSITY IN THE UNITED STATES AND A UNIVERSITY IN SAUDI ARABIA
}

\author{
Fahad Alfifi, Robert Morris University, faast219@mail.rmu.edu \\ Wenli Wang, Robert Morris University, wangw@rmu.edu \\ Gary Alan Davis, Robert Morris University, davis@rmu.edu \\ Paul J. Kovacs, Robert Morris University, kovacs@rmu.edu \\ Saeed Q. Al-Maliki, King Khalid University, salkhalidi@kku.edu.sa
}

\begin{abstract}
Cloud computing is similar to any new technology and can cause major challenges and opportunities for faculty in institutions of Higher Education. This study was undertaken to determine how Computer and Information Systems (CIS) faculty at two universities view the acceptance and use of cloud computing technology within their respective departments. One university is located in the United States and the other is located in Saudi Arabia. Specifically, this study sought to examine the commonalities and differences between these diverse universities as related to the advantages and disadvantages of using cloud computing, identify the key factors that influence adopting/using cloud computing, and determine each university's overall perception of cloud computing. This study also sought to determine if there are differences between CIS faculty in a U.S.-based university and in a Saudi Arabian-based university regarding perceived knowledge of Cloud Computing and the status of adoption of Cloud Computing
\end{abstract}

Keywords: Cloud Computing, Virtualization, Information Systems Education, Information Systems Faculty

\section{INTRODUCTION}

Cloud computing is an evolution and combination of several ideas that can be described as a new generation of computing that allows an organization to enhance its information technology capacity. Cloud computing technology combines virtualization, grid computing, and time-sharing, where the primary activities of data storage and computation shift to ever-bigger clusters of centrally-managed computers [3].

Cloud computing has different definitions, which are based on the different services being offered as well as the type and size of the organization At its broadest usage, cloud computing is the delivery of scalable Information Technology resources using the Internet, as opposed to hosting and operating those resources locally. These resources can include applications and services and the infrastructure on which they operate [5]. For example, by shifting computing needs to the cloud, an organization is able to replace an extensive and expensive computing infrastructure with services where costs are incurred on a per-usage basis. This means that the cost of computing services changes from a fixed-cost, to a variable cost.

The formal definition of cloud computing comes from The National Institute of Standards and Technology (NIST), which defines Cloud computing as " ... a model for enabling ubiquitous, convenient, on-demand network access to a shared pool of configurable computing resources (e.g., networks, servers, storage, applications, and services) that can be rapidly provisioned and released with minimal management effort or service provider interaction. This cloud model is composed of five essential characteristics, three service models, and four deployment models [16].

The essential characteristics of cloud computing are:

1. On-demand self-services: the consumer performs their necessary operations to obtain the needed services without human intervention with each service provider, such as email, applications, server and network storage.

2. Broad network access: consumers are able to work from anywhere at any time through standard mechanisms that promote use by heterogeneous thin or thick client platforms (e.g., mobile phones, tablets, workstations and laptops). 
3. Resource pooling: consumers are able to access a pool of services using the multi-tenant model that provides provisional and scalable services with different physical and virtual resources dynamically assigned and reassigned according to consumer demand. e.g., storage, processing, memory, and network bandwidth.

4. Rapid elasticity: consumers are able to access tremendous computing power, as well as have the ability to scale resources up and down, as needed or to scale rapidly outward and inward commensurate with demand.

5. Measured service. Cloud systems automatically control and optimize resource use by leveraging a metering capability at some level of abstraction appropriate to the type of service (e.g., storage, processing, bandwidth, and active user accounts). Resource usage can be monitored, controlled, and reported, providing transparency for both the provider and consumer of the utilized service [16].

Organizations and users can implement cloud computing through the three service models, which are: 1) Infrastructure as a Service, 2) Platform as a Service, and 3) Software as a Service [10, 14]. Infrastructure as a Service (IaaS) is the ability to provide full computer and information technology infrastructure resources, through the Internet, as "service-on-demand" [14]. IaaS provides services to organizations, such as a server, data storage, networking, operating systems, and application software. Platform as a Service (PaaS) is an application development environment that includes tools and operating systems that help users to manage and control infrastructure applications. PaaS uses online, in-cloud services, such as Microsoft's Windows Azure. Software as a Service (SaaS) is just an application delivered through the Internet on a "pay-as-you-go" basis, and as licensed services to end users over the Internet $[6,10,11]$.

Additionally, cloud computing can be described based on an organization's needs, level of security, level of trust, and amount of applicable regulations. In this context, there are four levels of cloud computing forms: 1) public cloud, 2) private cloud, 3) hybrid, and 4) community. In a public cloud, cloud computing is outsourced to a third party. In a private cloud, cloud computing is "insourced" (i.e., implemented internally within an organization), and in the hybrid model, cloud computing is made up of a combination of outsourced and "insourced" implementation. Finally, with the community cloud, the infrastructure is provisioned for sole use by a particular community of consumers from organizations that have shared concerns (e.g., security, mission, policy, requirements, and compliance considerations). Under the community scenario, the cloud may be owned, managed, and operated by one or more of the organizations in the community, a third party, or some combination of them, and it may exist on or off premises $[6,16]$.

Cloud computing technology appears to be an excellent solution for many universities that struggle with information technology (IT) complexity and cost. At the same time, cloud computing can provide universities with significant flexibility and agility [12]. Cloud computing, as observed in many university experiences, can handle many products and services that are provided by traditional IT departments (e.g., email services, operating systems, PCs, and servers). In addition, cloud computing can offer the IT developer many different tools and options to improve projects (e.g., web applications) [14].

\section{RESEARCH QUESTIONS}

This study was undertaken to determine how Computer and Information Systems (CIS) faculty at two institutions of higher education view the use of cloud computing technology. The study identified the internal and external factors that can influence CIS faculty in their use and adoption of cloud computing components.

Specifically, the study was conducted to answer the following research questions:

1. Among the four categories of survey questions concerning Cloud Computing, are there differences between the U.S.-based university and the Saudi Arabian-based university?

2. Are there differences between CIS faculty in a U.S.-based university and in a Saudi Arabian-based university regarding the perceived knowledge of Cloud Computing? 
3. Are there differences between CIS faculty in a U.S.-based university and in a Saudi Arabian-based university regarding the perceived status of adoption of Cloud Computing?

4. What is the role of culture differences that may contribute to the differences in the faculty perceptions between the U.S.-based university and the Saudi Arabian-based university?

\section{RELATED TRENDS AND STUDIES}

According to the CDW Corporations annual report [4], a survey of 1,242 information technology decision makers found that the number of universities that adopt or plan to adopt cloud computing has been increasing, compared to the annual report of 2011. The reasons indicated for this increase are as follows: $55 \%$ increase in efficiency, a $49 \%$ improvement in mobility, a $32 \%$ increase in the ability to innovate, a $31 \%$ increase in available IT staff for other projects, a $25 \%$ reduction in IT operating costs, and a $24 \%$ increase in the ability to offer new products/services. The CDW report also revealed that $43 \%$ of higher-education institutions are implementing or maintaining cloud computing. This number has increased by $10 \%$, compared to $34 \%$ in 2011 [4]. Finally, the CDW found that the specific applications that universities were moving to the Cloud are: 1) Storage (31\%), 2) Messaging/conferencing and collaboration (29\%), and 3) Computing power (25\%).

Cloud computing offers a variety of services that vary in importance from one organization to another. Most organizations are focused on providing enough cloud computing resources to satisfy users' demands, reduce upfront resource commitments, and allow customers to "pay-as-you-go" for services [2]. Universities are using cloud computing technology to replace traditional software by delivering applications and services through the Internet. The reasons behind the adoption of cloud computing are driven by reductions in Information Technology (IT) costs, reductions in systems complexity, and the opportunity to gain functionality that is more agile and flexible.

Many universities in the United States have started to adopt cloud computing, such as the University of California, Washington State University, the University of Washington, the University of Chicago, the University of California at Berkley, Cornell University, the University of Massachusetts at Amherst, and Carnegie Mellon University [14, 15]. For example, the University of California at Berkley moved one of its courses, which required a huge amount of servers, from their information technology department to cloud computing, hosted by Amazon Web services [7].

Cloud computing helps universities focus on core activities, such as research and innovations, rather than spending time and resources on complex information technology configuration and software systems. Katz [9] defined the most common features cloud computing offers to universities [9]. According to Katz [9] cloud computing ...

1. Increases access to scarce IT expertise and talent.

2. Scales IT services and resources.

3. Promotes further IT standardization.

4. Accelerates the time to market through IT supply bottleneck reductions.

5. Channels or counters the ad-hoc consumer of enterprise IT services.

6. Facilitates the transparent matching of IT costs, demand and funding.

7. Increases interoperability.

8. Supports a model of a $24 \times 7 \times 365$ environment.

9. Enables the sourcing of cycles and storage powered by renewable energy.

10. Drives down capital and total costs of IT in higher education.

The attractive aspects that are offered by cloud computing have influenced many universities to adopt and take full advantage of this technology. For example, North Carolina State University was able to reduce costs and information technology complexity. By outsourcing some in-house services to the cloud, North Carolina State University was able to reduce the campus information technology staff from 15 to 3 employees [17]. However, adoption levels of cloud computing in universities are still much lower than in the private, commercial, and governmental sectors [9].

Cloud computing in Saudi Arabia is still in early stages of use. However, an increasing number of Information Technology Organizations in Saudi Arabia are showing an interest in adopting Cloud computing. A report by IDC 
indicated that total spending on cloud delivery in Saudi Arabia had increased 34.86 percent in 2012, with long term spending to expand at a compound annual growth rate of 49.7 percent between 2012 and 2016 [13]. Additionally, although organizations in Saudi Arabia traditionally preferred to internally manage their IT operations, there has been interest in outsourcing models, with organizations gradually using hosting and managed services. This increasing acceptance of outsourcing services can be seen as an initial step toward progressing to a cloud-based model.

At the university-level in Saudi Arabia, Alhazzani [1] surveyed 200 faculty members at King Saud University to determine the advantages and disadvantages of cloud computing in education and to analyze the extent of using cloud computing tools by the faculty. The findings indicated that $56.7 \%$ of the respondents were familiar with the concept of cloud computing, $6.7 \%$ were neutral on the concept of cloud computing, and $3.3 \%$ are strongly familiar with the concept of cloud computing. The entire sample agreed that the concept of cloud computing provided access to applications from anywhere at any time. The respondents also agreed that cloud computing is easy to use, and involves low maintenance.

The findings by Alhazzani also indicated that there is an agreement among the respondents on the disadvantages of cloud computing, in terms of concerns about data storage on other servers. Many of the respondents were also concerned about publishing policy and property rights, and on the stability and security of essential data. Administrative and infrastructure constraints and financial constraints were also concerns. Finally, the research findings indicated that the vast majority of the respondents $(96.7 \%)$ agreed that the idea of cloud computing education is certainly a huge step towards the development of a Higher Education system in Saudi Arabia.

\section{METHODS AND PROCEDURES}

The current study involved the administration of an online survey to Computer and Information Systems (CIS) faculty at two universities. One university is a public institution located in the Asir Province in the southwest region of Saudi Arabia, and the other university is a private institution located in the United States (Pennsylvania). Of the 42 total CIS faculty members from both universities, 40 (95.24\%) completed the survey. More specifically, 19 CIS faculty from the university in the United States, and 21 CIS faculty from the university in Saudi Arabia completed the survey.

The survey, which consisted of 29 closed-ended questions, was created and administered using QuestionPro Online Survey Software, from QuestionPro, Inc. The 29 survey questions were divided into four major categories. These four categories were: 1) Faculty perceptions regarding Cloud Computing (five questions), 2) Perceived advantages of using Cloud Computing (seven questions), 3) Perceived disadvantages of using Cloud Computing (seven questions), and 4) Key factors that influence the adoption of Cloud Computing (seven questions). Each question, within each category, is shown in APPENDIX A. These questions used Likert-like scales with descriptors of five steps or levels of agreement/disagreement. The Likert items were balanced on both sides of a neutral option, creating a less biased measurement. Specifically, the Likert levels for these questions were Strongly Agree (value $=5$ ), Fairly Agree (value $=4)$, Neutral $($ value $=3)$, Partly Disagree $($ value $=2)$, and Strongly Disagree $($ value $=1)$.

In addition to the above-mentioned four categories and 26 questions, the survey asked respondents three additional questions. These questions were: 1) Indicate your perceived knowledge of Cloud Computing, 2) Indicate the status of Cloud Computing within your university's CIS department, and 3) What services or applications should be migrated to the cloud? For this study, only the preceding questions 1 and 2 were studied with the following levels of agreement/disagreement:

Question 1 - Indicate your perceived knowledge of Cloud Computing: Knowledgeable (value = 5), Very knowledgeable (value $=4)$, Somewhat knowledgeable (value $=3$ ), Not very knowledgeable (value $=2)$, and No knowledge $($ value $=1)$.

Question 2 - Indicate the status of Cloud Computing within their university's CIS department: Maintaining (value = 5), Implementing (value $=4$ ), Planning (value $=3$ ), Discovery (value $=2$ ), and Not Considering (value $=1$ ). 
Once collected, all survey results were analyzed using SPSS (Statistical Package for the Social Sciences). Descriptive statistics were generated in SPSS to calculate the participants' mean rating scores, as related to Cloud Computing. In addition, the Independent Samples T-Test was used to determine if any noted differences in mean rating scores between CIS faculty at the U.S-based university and Saudi Arabian-based university were statistically significant.

\section{RESEARCH RESULTS}

This section presents the findings of the survey participants' responses to the four research questions. An Independent Samples T-Test was used to determine if any noted differences in mean rating scores between CIS faculty at the U.S-based University and Saudi Arabian-based University were statistically significant. The results for each research question are reported in tabular format.

The first research question sought to determine if there were differences, among the four categories of Cloud Computing survey questions, between the U.S-based university and Saudi Arabian-based university. As stated previously, the four categories were: 1) Faculty perceptions regarding Cloud Computing, 2) Perceived advantages of using Cloud Computing, 3) Perceived disadvantages of using Cloud Computing, and 4) Key factors that influence the adoption of Cloud Computing. Out of the four categories above, the only category that resulted in a statistically significant difference between the two universities was the fourth category (i.e., Key factors that influence the adoption of Cloud Computing). Within this category, the results to five of the seven questions showed a statistically significant difference at $p<.05$ and two questions did not reach statistical significance. The Independent Samples T-Test Results of these seven questions from this category are listed in Table 1.

Table 1: Independent Samples T-Test Results of Factors that Influence the Adoption of Cloud Computing

\begin{tabular}{|c|c|c|c|c|c|c|c|}
\hline Question & $\begin{array}{c}\text { U.S.-based } \\
\text { University } \\
\text { Mean }\end{array}$ & $\begin{array}{l}\text { U.S.- } \\
\text { based } \\
\text { Universi } \\
\text { ty Std. } \\
\text { Dev. }\end{array}$ & $\begin{array}{c}\text { Saudi- } \\
\text { based } \\
\text { University } \\
\text { Mean }\end{array}$ & $\begin{array}{c}\text { Saudi- } \\
\text { based } \\
\text { University } \\
\text { Std. Dev. }\end{array}$ & $\mathbf{t}$ & df & Sig. \\
\hline $\begin{array}{l}\text { Cloud Computing } \\
\text { enables me to } \\
\text { accomplish tasks } \\
\text { more quickly. }\end{array}$ & 3.68 & .946 & 4.48 & .680 & 3.013 & 1,38 & $.005^{* *}$ \\
\hline $\begin{array}{l}\text { Cloud Computing } \\
\text { enhances my } \\
\text { effectiveness on the } \\
\text { job. }\end{array}$ & 3.26 & .806 & 4.14 & .793 & 3.475 & 1,38 & $.001 * *$ \\
\hline $\begin{array}{l}\text { Cloud computing } \\
\text { makes it easier to } \\
\text { do my job. }\end{array}$ & 3.37 & .761 & 4.33 & .913 & 3.643 & 1,38 & $.001 * *$ \\
\hline $\begin{array}{l}\text { Cloud computing } \\
\text { tools and } \\
\text { applications are } \\
\text { flexible. }\end{array}$ & 3.63 & .895 & 4.33 & .730 & 2.700 & 1,38 & $.011^{*}$ \\
\hline $\begin{array}{l}\text { I could easily } \\
\text { become skillful } \\
\text { with Cloud } \\
\text { Computing tools } \\
\text { and applications. }\end{array}$ & 4.00 & .816 & 4.57 & .598 & 2.504 & 1,38 & $.017^{*}$ \\
\hline
\end{tabular}




\begin{tabular}{|c|c|c|c|c|c|c|c|}
\hline $\begin{array}{l}\text { I would be willing } \\
\text { to learn to use } \\
\text { cloud computing } \\
\text { services, } \\
\text { application and } \\
\text { tools }\end{array}$ & 4.37 & 1.065 & 4.67 & 0.73 & 1.961 & 1,38 & .17 \\
\hline $\begin{array}{l}\text { Using the cloud } \\
\text { computing } \\
\text { technology in my } \\
\text { job increase my } \\
\text { productivity }\end{array}$ & 3.63 & 0.955 & 4.38 & 0.669 & 3.23 & 1,38 & $.08^{*}$ \\
\hline
\end{tabular}

The second research question sought to determine if there were differences between CIS faculty in a U.S.-based university and in a Saudi Arabian-based university regarding the perceived knowledge of Cloud Computing. The results from the seven survey questions are summarized in Table 2.

As the results in Table 2 indicate, the CIS faculty from the Saudi-based university reported that they had a higher level of knowledge than the CIS faculty from the U.S.-based university, regarding cloud computing $(\bar{x}=4.14)$. However, the difference in means was not statistically significant at the .05 level $(\mathrm{t}=-1.839, \mathrm{p}=.074)$. The mean scores regarding knowledge of cloud computing are listed in Table 2.

Table 2: Independent Samples T-Test Results for Cloud Computing Perceived Knowledge

\begin{tabular}{|l|c|c|c|c|c|}
\hline & Mean & Std. Dev. & t-test & df & Sig. \\
\hline U.S.-Based University & 3.74 & .734 & -1.839 & 1,38 & .074 \\
\hline $\begin{array}{l}\text { Saudi-Based } \\
\text { University }\end{array}$ & 4.14 & .655 & & & \\
\hline
\end{tabular}

The third research question sought to determine if there were differences between CIS faculty in a U.S.-based university and in a Saudi Arabian-based university regarding the perceived status (or level of adoption) of Cloud Computing. As previously stated, the five levels of cloud computing adoption were Maintaining (value $=5$ ), Implementing (value $=4$ ), Planning (value $=3$ ), Discovery (value $=2$ ), and Not Considering (value $=1$ ). As the results in Table 3 indicate, the CIS faculty from the U.S.-based university reported that they are further along in their adoption status of cloud computing technology than CIS faculty at the Saudi Arabian-based university $(\bar{x}=3.16)$. However, the difference in means was not statistically significant at the .05 level $(\mathrm{t}=2.018, \mathrm{p}=.051)$. The mean scores regarding level of cloud computing status are listed in Table 3.

Table 3: Independent Samples T-Test Results for Cloud Computing Status Within Your Department

\begin{tabular}{|l|c|c|c|c|c|}
\hline & Mean & Std. Dev. & t-test & df & Sig. \\
\hline U.S.-Based University & 3.16 & 1.302 & 2.018 & 1,38 & .051 \\
\hline $\begin{array}{l}\text { Saudi-Based } \\
\text { University }\end{array}$ & 2.33 & 1.278 & & & \\
\hline
\end{tabular}

The fourth research question attempted to determine what the role culture differences may contribute to the differences in the faculty perceptions between the U.S.-based university and the Saudi Arabian-based university? In Category 4, regarding the key factors that Influence the Adoption of Cloud Computing, for six out of seven questions (See Table 1), the mean ratings from the CIS faculty in the Saudi-Arabian-based university are 
significantly higher than those from the U.S.-based university. For perceived knowledge (Table 2), the mean rating from the CIS faculty in the Saudi-Arabian-based university is also significantly higher $(\bar{x}=4.14)$ than that from the U.S.-based university $(\bar{x}=3.74)$. However, for the adoption category (Table 3$)$, the mean rating from the Saudibased CIS faculty is significantly lower $(\bar{x}=2.33)$ than that from the U.S.-based CIS faculty $(\bar{x}=3.16)$, indicating that the Saudi-based CIS department was at the "Discovery" level of adoption, while the U.S.-based CIS department was already at the "Planning" level of adoption. Interestingly, among the eight out of nine reported questions above, the standard deviations from the U.S.-based university are higher than those from the Saudi-based university.

Possible explanations of the differences in the means and standard deviations may come from the cultural differences of these two countries. According to Figure 1, generated by the Hofstede Center Web site [8], the Power-distance dimension of the Saudi Arabia is much higher (95) than that of the U.S. (40). This dimension deals with the fact that all people in societies are not equal, and that individuals accept a hierarchical order in which everyone has a place. This may explain why the mean ratings from the Saudi-based CIS faculty are generally higher than those from the U.S.-based CIS faculty-CIS faculty in Saudi Arabia have more optimistic views on the key factors influencing the adoption of a new technology; even when they have limited exposure or hands-on experiences with that technology.

In fact, the high Uncertainty-avoidance of the Saudi Arabia (80), in comparison to that of the U.S. (46) may help explain why the CIS faculty in Saudi Arabia took a slower pace of actually adopting the new technology than that of the CIS faculty in the U.S. CIS Faculty in Saudi Arabia are more cautious in taking actions despite the optimistic perceptions of the technology. The Individualism dimension of the U.S. is much higher (91) than that of the SaudiArabia (25), which may explain why the standard deviations are higher from U.S.-based CIS faculty — CIS faculty in the U.S. has more diverse views on these eight questions.

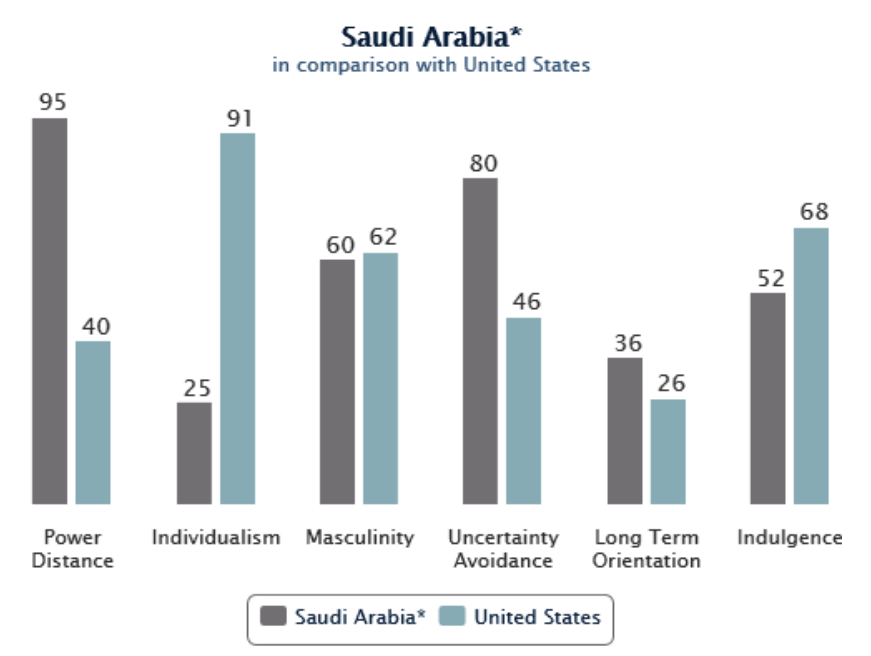

Figure 1. Saudi Arabia in Comparison with the United States [8].

\section{CONCLUSIONS}

Cloud computing is largely an evolution of Information Technology (IT) that is maturing from a promising business concept to one of the fastest growing segments of the IT industry. Moving academic IT services to the cloud may involve a formidable decision, since the use of cloud computing can change the sourcing and delivery of solutions. The current study was based on the views of 40 faculty members in the Computer and Information Systems (CIS) departments of two universities. One university is located in Pennsylvania, and the other university is located in the Southwest region of Saudi Arabia. These two institutions not only differ in geography, but also in language, culture, climate, economics, and political structure. However, it should be noted that since the sample is composed of 19 CIS faculty from the university in the United States and 21 CIS faculty from the university in Saudi Arabia and an onlines questionnaire was used, the results of this research may be limited and not generalizable to other CIS faculty and universities in the United States and Saudi Arabia. 
The CIS faculty from the Saudi-based university felt that they would derive greater benefits from the use of cloud computing than the U.S.-based CIS faculty. The Saudi-based CIS faculty also felt that they had a greater knowledge of cloud computing than their U.S.-based counterparts. However, these two findings must be examined in light of the third research question that indicated that U.S.-based CIS faculty is further along in the adoption of cloud computing services. Specifically, the U.S.-based CIS faculty indicated that they were at the "Planning" level of adoption, while the Saudi-based CIS faculty indicated that they were only at the "Discovery" level of adoption. Therefore, the perceptions of the Saudi-based CIS faculty may be premature, given their emerging level of cloud computing adoption.

The role of culture differences between the Saudi Arabia and the U.S. may explain the significant differences in the faculty perceptions between the U.S.-based university and the Saudi Arabian-based university regarding the key factors influencing the adoption of cloud computing, perceived knowledge of cloud computing, and the cloud computing status in the Computing Departments. The high power-distance in Saudi Arabian culture may explain why CIS faculty there have more optimistic views on the key factors influencing the adoption of a new technology, even when they have limited exposure or hands-on experiences with that technology. The high uncertaintyavoidance in Saudi Arabian culture may explain why CIS faculty there made slower progress in technology adoption despite the optimistic views on the technology. The high individualism in the United States culture may explain why in CIS faculty there have more diverse views.

Although there were no significant differences found between the CIS Faculty at the two universities concerning the perceived disadvantages of using Cloud Computing, a strong majority of the respondents expressed a concern that adopting cloud computing technology would increase security risks of proprietary data and applications. This finding is not surprising, given that security is a universal concern that crosses geographic, linguistic, cultural, economic, and political boundaries.

Finally, the responses by the CIS faculty at both universities were positive and view cloud computing as an attractive technology option for a CIS department. In addition, faculty from both universities were willing to learn cloud computing services, applications, and tools. The above conclusions are not unexpected, given that the CIS discipline involves the investigation, understanding, and use of new technologies. As a possible follow-up study, additional faculty could be surveyed to determine if the above conclusions can be generalized to other academic disciplines.

\section{REFERENCES}

1. Alhazzani, N. (2014) A proposed plan to use cloud computing in higher education at the Kingdom of Saudi Arabia, ICERI2014 Proceedings, p. 2895.

2. Armbrust, M., Fox, A., Griffith, R., Joseph, A. D., Katz, R. H., Konwinski, A., . . Zaharia, M. (2009). Above the clouds: A Berkeley view of cloud computing, Technical Report No. UCB/EECS-2009028. Available: http://www.eecs.berkeley.edu/Pubs/TechRpts/2009/EECS-2009-28.pdf

3. Baker, S. (2007, Dec 24) Google and the wisdom of clouds, Business Week.

4. CDW's 20130 State of the Cloud Computing Report, CDW LLC. Retrieved January 28, 2015 from http:/www.cdwnewsroom.com/wp-content/uploads/2013/02/CDW_2013_State_of_The_Cloud_Report_ Report_021113_FINAL.pdf

5. Educause, (2009, August). 7 things you should know about cloud computing. Available: http://net.educause.edu/ir/library/pdf/EST0902.pdf

6. Etsebeth E. (2012). Trialability, perceived risk and complexity of understanding as determinants of cloud computing services adoption. University of Pretoria. Available: http://repository.up.ac.za/bitstream/handle/2263/29623/dissertation.pdf?sequence=1\&isAllowed=y

7. Fox, A. (2009). Cloud computing in education. Available: https://inews.berkeley.edu/articles/Spring2009/cloudcomputing

8. Hofstede, Greet (2015), Saudi Arabia in comparison with the United States. Available: http:/geerthofstede.com/saudi-arabia.html

9. Katz, R. N., Goldstein, P. J. \& Yanosky, R. (2009). Demystifying cloud computing for higher education. EDUCAUSE Center for Applied Research Bulletin, 19, 1-13.

10. Leavitt, N. (2009). Is cloud computing really ready for prime time, 42(1), 15-20. 
11. Limbasan, Adriana. (2011). Implementing SaaS Solution for CRM. Informatica Economica. Available: $\mathrm{http}: / /$ www.readperiodicals.com/201104/2444603581.html

12. Nicholson J. (2009). Cloud computing's top issues for higher education. University Business. Available: http://www.universitybusiness.com/article/cloud-computings-top-issues-higher-education

13. Saudi Gazette, (2013), March), Saudi firms explore cloud computing to reduce costs. Available: $\mathrm{http} / /$ www.saudigazette.com.sa/index.cfm?method=home.regcon\&contentid=20130310156139.

14. Sultan, N. (2010). Cloud computing for education: A new dawn? International Journal of Information Management, 30, 109-116

15. Daly, J. (2009, April). The state of cloud computing in higher education. New survey highlights key opportunities for colleges. EdTech Focus on Higher Education. Available: http://www.edtechmagazine.com/higher/article/2013/02/state-cloud-computing-higher-education

16. Mell, P., \& Grance, T. (2011). The NIST definition of cloud computing. National Institute of Standards and Technology. Available: http://csrc.nist.gov/publications/nistpubs/800-145/SP800-145.pdf

17. Wyld, D. C. (2011). Cloud computing 101: Universities are migrating to the cloud for functionality and savings. Available: http://rolandstuart615.wordpress.com/2011/10/13/cloud-computing- 101-universities-aremigrating-to-the-cloud-for-functionality-and-savings/ 


\section{APPENDIX A}

\section{Four Major Categories of Questions}

Category 1. What is the perception of Computer and Information Systems faculty regarding cloud computing?

1. Cloud computing is an attractive technology option for a CIS department.

2. Increase the efficiency and quality of services.

3. I recommended adopting cloud technology in the CIS department.

4. Is an attractive economic option for a CIS department?

5. Increase data information security.

Category 2. What are the advantages of using cloud computing in your CIS department?

1. Accessing data/applications from anywhere/any time using any device Improved Mobility for Users

2. Improved Mobility for Users and provide more storage capacity/share data/email.

3. Provide more storage capacity/share data/email.

4. Improved data backup /disaster recovery

5. Reduced IT complexity/workload

6. Provide working scalability/flexibility environment Increased cost savings

7. Increased cost savings

Category 3. What are the disadvantages of using cloud computing in CIS department?

1. Lack of standards among cloud service providers

2. Concerns with security of proprietary data/ applications

3. Integration with current networks/applications/systems

4. System performance of cloud services

5. Legal and regulatory compliance

6. Lack of reliability

7. Lack of availability

Category 4. What are the key factors that influence CIS faculty in relation to adopting/using cloud computing?

1. I would be willing to learn to use cloud computing services, application and tools.

2. It would be easy for me to become skillful at using the cloud computing application and tools.

3. Enables me to accomplish tasks more quickly.

4. Increases my productivity in my job.

5. Makes it easier to do my job

6. Cloud computing applications and tools would be flexible with which to interact.

7. Enhances my effectiveness on the job. 